\title{
When all reduced strongly flat modules are projective
}

\author{
LÁszló Fuchs (*) - SAng Bum Lee (**)
}

ABstRACT - We consider integral domains over which all the reduced strongly flat modules are projective, and give several characterizations of such domains (Theorem 1.1). Those $h$-local Prüfer-domains are identified which share this property (Theorem 3.3).

Mathematics Subject Classification (1991). 13C05, $13 \mathrm{G} 05$.

KEYWORDs. Projective, strongly flat, Matlis-cotorsion modules, self-small module.

\section{Introduction}

Throughout, $R$ will denote an integral domain that is not a field, $Q$ its field of quotients and $K=Q / R$.

Strongly flat modules have received recently a lot of attention in the theory of modules over integral domains. Their importance lies primarily in the fact that the classes of strongly flat and Matlis-cotorsion modules form a so-called cotorsion pair (see e.g. Göbel-Trlifaj [6]). Strong flatness was introduced by Bazzoni-Salce [2]: a module $S$ is called strongly flat if $\operatorname{Ext}_{R}^{1}(S, M)=0$ holds for all Matlis-cotorsion modules $M$. (Recall: an $R$-module $M$ is said to be Matlis-cotorsion, if it satisfies $\operatorname{Ext}_{R}^{1}(Q, M)=0$.) They proved that a module is strongly flat if and only if it is a direct summand of a module $N$ fitting in a short exact sequence

$$
0 \rightarrow F \rightarrow N \rightarrow D \rightarrow 0
$$

(*) Indirizzo dell'A.: Department of Mathematics, Tulane University, New Orleans, Louisiana 70118, USA.

E-mail: fuchs@tulane.edu

(**) Indirizzo dell'A.: Department of Mathematics, Sangmyung University, Seoul 110-743, Korea.

E-mail: sblee@smu.ac.kr 
where $F$ is a free and $D$ is a torsion-free divisible $R$-module. Such an $N$, if reduced, lies between the free module $F$ and its $R$-completion $\widetilde{R}$; this follows from the natural isomorphism $\operatorname{Hom}(N, \widetilde{F}) \cong \operatorname{Hom}(F, \widetilde{F})$ that is a consequence of the standard Hom-Ext exact sequence. (Reduced means that it contains no divisible submodule $\neq 0$.)

Bass [1] characterized the (not necessarily commutative) rings over which flat modules are projective: these are the perfect rings. In this note, our main objective is to deal with an analogous natural, but less ambitious question for strongly flat modules: which are the domains over which all reduced strongly flat modules are projective? (The restriction to domains is necessary, as so far strong flatness has been defined only for domains.)

Interestingly, this question turns out to be related to the existence of certain test modules.

The investigations in Fuchs-Göbel [3] were concerned with domains over which reduced test modules exist for the property of being Matliscotorsion. By definition, $U$ is such a test module if $\operatorname{Ext}_{R}^{1}(U, M)=0$ implies that $M$ is Matlis-cotorsion. As the classes of strongly flat and Matlis-cotorsion modules form a cotorsion pair, only the reduced strongly flat modules are of real interest as test modules. It was shown in [3] that no such test module exists that would work for modules of all sizes. However, if the modules $M$ under consideration are of bounded cardinality $\kappa$ (no matter how large we choose $\kappa$ ), then such a test module (a strongly flat one) can be found exactly if the module $K$ is not self-small. Here a module $M$ is called self-small if the image of a homomorphism $M \rightarrow \oplus_{\kappa} M$ (with arbitrary cardinal $\kappa$ ) is contained in a finite direct sum. (It is rather obvious that it suffices to require this property only for $\kappa=\aleph_{0}$.)

This note is devoted to proving the following theorem that furnishes us with relevant information about these domains.

THEOREM 1.1. For a domain $R$ that is not a field, the following conditions are equivalent:

(i) reduced strongly flat $R$-modules are projective;

(ii) $R$ is complete and there are no reduced test modules for Matliscotorsionness;

(iii) $R$ is complete and the module $K$ is self-small;

(iv) projective $R$-modules are Matlis-cotorsion;

(v) strongly flat $R$-modules are Matlis-cotorsion. 
Note that in (iv)-(v), "Matlis-cotorsion" can be replaced by " $R$-complete", because a reduced torsion-free module is Matlis-cotorsion if and only if it is $R$-complete. The proof of the theorem will be given below via the implications (i) $\Rightarrow$ (ii) $\Leftrightarrow$ (iii) $\Rightarrow$ (iv) $\Rightarrow$ (v) $\Rightarrow$ (i).

We wish to thank the referee for valuable comments.

\section{Lemmas and proof of theorem}

We start with preliminary lemmas. The first lemma shows that the completion of a domain can be a projective module only in the obvious case.

LEMMA 2.1. Let $R$ be a domain with the property that the $R$-completion $\widetilde{R}$ of $R$ is a projective $R$-module. Then $R=\widetilde{R}$.

Proof. Choose a non-unit $r \neq 0$ in $R$, and consider the diagram

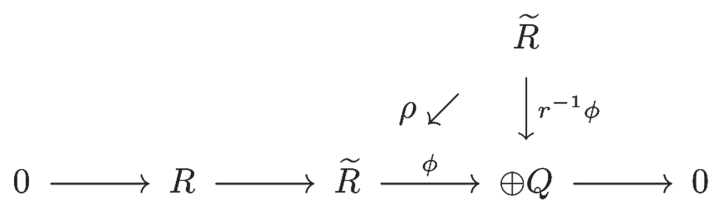

where the map $\rho: \widetilde{R} \rightarrow \widetilde{R}$ exists making the diagram commute because $\widetilde{R}$ is projective by hypothesis. $\rho$ is an endomorphism of $\widetilde{R}$, so it acts as multiplication by an element $s \in \widetilde{R}$. Thus for every $x \in \widetilde{R}$ we have $\phi(s x)=r^{-1} \phi(x)$. Letting $x=1$, we see that $s$ must be an element of $R$, so we can write $r s \phi(x)=\phi(x)$ in $\oplus Q$. If $\phi(x) \neq 0$ for some $x \in \widetilde{R}$, then we must have $r s=1$, which cannot happen unless $r \in R$ is a unit. Therefore, the direct sum $\oplus Q$ must be 0 , i.e. $R=\widetilde{R}$.

Actually, even a stronger result can be stated: if $\widetilde{R}$ is contained in a projective $R$-module, then $R=\widetilde{R}$. In fact, it is shown in Lee [7] that strongly flat submodules of projective modules over any domain are themselves projective. This theorem along with our preceding lemma justifies our claim.

Since [7] has not been published as yet, we sketch a short proof of the cited result. Let $F$ be a strongly flat submodule in a projective $R$-module $P$. To show that $F$ is projective, it suffices to prove that p.d. $P / F \leq 1$ which will be done by showing that $\operatorname{Ext}_{R}^{1}(P / F, D)=0$ for all $h$-divisible torsion modules $D$ (cf. [4, Proposition 2.5, p. 252]). For such a $D$, we form the exact 
sequence $0 \rightarrow M \rightarrow \oplus Q \rightarrow D \rightarrow 0$ where $M$ is a Matlis-cotorsion module (see Matlis [8]). In view of the exact sequence

$$
0=\operatorname{Ext}_{R}^{1}(P / F, \oplus Q) \rightarrow \operatorname{Ext}_{R}^{1}(P / F, D) \rightarrow \operatorname{Ext}_{R}^{2}(P / F, M)
$$

it will be enough to show that $\operatorname{Ext}_{R}^{2}(P / F, M)=0$. In the exact sequence

$$
\operatorname{Ext}_{R}^{1}(F, M) \rightarrow \operatorname{Ext}_{R}^{2}(P / F, M) \rightarrow \operatorname{Ext}_{R}^{2}(P, M)=0,
$$

induced by the exact sequence $0 \rightarrow F \rightarrow P \rightarrow P / F \rightarrow 0$, we have $\operatorname{Ext}_{R}^{1}(F, M)=0$ as $F$ is strongly flat. Thus $\operatorname{Ext}_{R}^{2}(P / F, M)=0$, and the projectivity of $F$ follows.

We state the following result without proof; for a proof see the reference.

LEMma 2.2 (Fuchs-Göbel [3]). Let $R$ be any domain. For $R$-modules of bounded cardinality there exist reduced strongly flat test modules if and only if $K=Q / R$ is not self-small.

The proof of our next lemma is borrowed from Fuchs-Salce [4, p. 281].

LEMMA 2.3. Let $R$ be a domain complete in its $R$-topology. If the module $K=Q / R$ is self-small, then free $R$-modules are Matlis-cotorsion.

Proof. In view of the Matlis category equivalence, the $R$-completion of a free $R$-module $F=\oplus_{\kappa} R$ may be given by

$$
\widetilde{F} \cong \operatorname{Hom}_{R}\left(K, K \otimes_{R} F\right)=\operatorname{Hom}_{R}\left(K, \oplus_{\kappa}\left(K \otimes_{R} R\right)\right)=\operatorname{Hom}_{R}\left(K, \oplus_{\kappa} K\right) .
$$

If $K$ is self-small, then the last Hom is $\oplus_{\kappa} \operatorname{Hom}_{R}(K, K)=\oplus_{\kappa} \widetilde{R}=F$, i.e. free modules are Matlis-cotorsion.

Proof of Theorem. (i) $\Rightarrow$ (ii): The completeness of $R$ follows from Lemma 2.1, and clearly, projective modules cannot be test modules.

(ii) $\Leftrightarrow$ (iii): This equivalence follows at once from Lemma 2.2.

(iii) $\Rightarrow$ (iv): See Lemma 2.3.

(iv) $\Rightarrow$ (v): If (iv) holds, then in (1) both $F$ and $D$ are Matlis-cotorsion. Therefore, the same holds for $N$.

(v) $\Rightarrow$ (i): If the module $N$ in (1) is reduced, then it has the same Matlis-cotorsion envelope as $F$. Therefore, if (v) holds, then $F=N$ must be Matlis-cotorsion. We conclude that (i) holds. 
Next we exhibit two examples for domains that satisfy the conditions listed in Theorem 1.1.

EXAMPLE 2.4. Let $R$ be a complete valuation domain such that p.d. $Q>1$; this hypothesis on $Q$ is equivalent to saying that it is uncountably generated. Then $K$ is self-small as is easy to see (shown e.g. in [4, p. 281]).

EXAMPLE 2.5 ([3, p. 88]). Let $R=Z[X]$ be the polynomial ring with an uncountable set $X$ of indeterminates. Göbel-May [5, Example 3.8] proved that $R$ is complete in the $S$-topology, where $S$ denotes the set of monomials in $R$. It is easily seen that the same holds for free $R$-modules. Then free $R$-modules are also $R$-complete, since the $R$-topology is finer than the $S$-topology.

\section{Additional results}

From the following lemma it will be clear that no genuine Matlis domain may have the properties listed in Theorem 1.1. (Recall: Matlis domains $R$ are defined by the condition that p.d.Q $\leq 1$.)

Lemma 3.1. Let $R$ be a Matlis domain with the property that free modules are $R$-complete. Then $R$ is a field.

Proof. Suppose $R$ is as stated, and $N$ is an arbitrary $R$-module. There exists an exact sequence $0 \rightarrow H \rightarrow F \rightarrow N \rightarrow 0$ with a free module $F$. We get the induced exact sequence

$$
0=\operatorname{Ext}_{R}^{1}(Q, F) \rightarrow \operatorname{Ext}_{R}^{1}(Q, N) \rightarrow \operatorname{Ext}_{R}^{2}(Q, H)=0
$$

where the first Ext is 0 because $F$ is $R$-complete and the third Ext vanishes because p.d. $Q=1$. This shows that $Q$ is projective, thus $R$ is a field.

We turn our attention to $h$-local domains.

LEMMA 3.2. Let $R$ be an h-local domain satisfying the conditions of Theorem 1.1. Then

(i) $R$ is semilocal, and

(ii) every localization $R_{P}$ at a maximal ideal $P$ also satisfies these conditions. 
Proof. (i) If $R$ is not semilocal, then in the decomposition of $K$ into the direct sum of its $P$-components (for maximal ideals $P$ ) there are infinitely many non-zero components. Such a $K$ cannot be self-small.

(ii) If $R$ is $h$-local, then $Q / R_{P}$ is a summand of $Q / R$, so it inherits selfsmallness.

We can now derive the following corollary:

THeOREm 3.3. An h-local Prüfer-domain $R$ satisfies the conditions listed in Theorem 1.1 if and only if it is complete, semilocal, and each of its localizations at maximal ideals has an uncountably generated quotient field.

Proof. This follows easily from the above considerations.

In particular, a valuation domain satisfies the conditions of Theorem 1.1 if and only it is of the kind described in Example 2.4.

We would like to point out that the problem becomes trivial if we look for domains over which all flat modules are Matlis-cotorsion. In fact, we have:

LEMMA 3.4. The only integral domains over which all flat modules are Matlis-cotorsion are the fields.

Proof. For an arbitrary module $N$ consider the exact sequence $0 \rightarrow H \rightarrow F \rightarrow N \rightarrow 0$ where $F$ is the flat cover of $N$ and $H$ is Enochs-cotorsion (i.e. $\operatorname{Ext}_{R}^{1}(G, H)=0$ for all flat modules $G$ ). We get the induced exact sequence (2) where the first Ext is 0 , since $F$ is Matlis-cotorsion, and the third Ext vanishes, since $H$ is Enochs-cotorsion. Thus $Q$ is projective, and the domain is a field.

A final remark may be inserted here that concerns cotorsion pairs. As mentioned above, the classes of strongly flat and Matlis-cotorsion modules form a cotorsion pair, i.e. $\operatorname{Ext}_{R}^{1}(S, M)=0$ for all strongly flat $S$ and all Matlis-cotorsion $M$, and in addition, these are the largest classes with this property. Now, our Theorem 1.1 characterizes the case when the class of strongly flat modules is contained in the class of Matlis-cotorsion modules. It also gives full information about the case when all projective modules are in the kernel of this cotorsion pair.

The dual question when all torsion-free Matlis-cotorsion modules are strongly flat can be answered easily for Matlis domains: this is the case if 
and only if the Matlis domain is Dedekind. In fact, over Dedekind domains all torsion-free modules are strongly flat. On the other hand, the Matlis category equivalence yields the exact sequence $0 \rightarrow M \rightarrow \oplus Q \rightarrow D \rightarrow 0$ for every $h$-divisible $D$ with $M$ torsion-free Matlis-cotorsion. If $M$ is strongly flat, then $D$ will have strongly flat dimension $\leq 1$, so also projective dimension $\leq 1$ (see Lee [7, Theorem 4.5]). A domain whose $h$-divisible modules have p.d. $\leq 1$ is Dedekind.

\section{REFERENCES}

[1] H. BASs, Finitistic dimension and a homological generalization of semiprimary rings, Trans. Amer. Math. Soc. 95 (1960), 466-488.

[2] S. BAzzoni and L. SAlce, Almost perfect domains, Coll. Math. 95 (2003), 285301.

[3] L. Fuchs and R. GöBEL, Testing for cotorsionness over domains, Rendiconti Sem. Mat. Univ. Padova 118 (2007), 85-99.

[4] L. Fuchs and L. SAlce, Modules over non-Noetherian Domains, Math. Surveys and Monographs, vol. 84 (Amer. Math. Society, Providence, 2001).

[5] R. GöBEL and W. MAY, Independence in completions and endomorphism algebras, Forum Math. 1 (1989), 215-226.

[6] R. GöBel and J. TRLifaJ, Approximations and Endomorphism Algebras of Modules, Expositions in Math., vol. 41 (W. de Gruyter, 2006).

[7] S.B. LEE, Strongly flat modules over Matlis domains (submitted).

[8] E. Matlis, Cotorsion modules, Memoirs Amer. Math. Soc. 49 (1964).

Manoscritto pervenuto in redazione il 27 Aprile 2012. 
